\title{
Proto-MIP - A novel route for MIP fabrication
}

\author{
Sandra Haas ${ }^{1}$, Leo Schranzhofer ${ }^{1}$ \\ 1 Profactor GmbH, Im Stadtgut A2; 4407 Steyr-Gleink \\ Sandra.haas@profacotr.at, leo.schranzhofer@profactor.at
}

\begin{abstract}
Molecular Imprinting is a method to synthesize materials with biomimetic properties that can be used in sensor systems or assays replacing their biological equivalents like antibodies or cells. Due to the self assembly step and polymerization chemistry, MIPs bear the drawback of low fabrication reproducibility of $20-50 \%$, a reason for MIPs being less used in applications. Proto-MIP is aimed to a spatially separated self-assembly process during generation of an imprinted layer of anchor groups around an immobilized template, like bacteria or molecules. This is followed by merging this anchor group layer with an UV-curable resin to generate a full MIP layer within seconds. Yielding an imprinted layer resembling shape, size and complementary chemical information of the template, which is exhibiting an affinity of high sensitivity and specificity towards the respective antigen or analyte. The adaption of MIP fabrication to UV-NIL processes enables improved batch fabrication and reduction of fabrication time and costs.
\end{abstract}

Key words: molecularly imprinted polymers, self-assembly, functional monomers, E.coli

\section{Introduction}

Molecularly imprinted polymers (MIPs) is a concept to generate highly selective cavities within a polymer network. Briefly, functional monomers self-assembly around a template molecule/particle. On polymerization, followed by extraction of the template, a geometrical and chemical pattern is left behind. The produced MIPs constitute artificial recognition structures exhibiting significant affinity towards their respective analyte. They are considerably more robust, stable and withstand a broader range of conditions than their biological counterparts such as antibodies or enzymes. In addition, molecular imprinting is a straight-forward technique to synthesize surfaces with biomimetic properties. Moreover, the generation of such artificial binding sites is highly cost effective and less resource-demanding [1].

However, MIP fabrication currently is lacking in batch-to-batch as well as selectivity and reproducibility due to the necessary selfassembly process [2]. The here described ProtoMIP approach is dealing with increasing reproducibility and decreasing fabrication times as well as taking the next step in bringing MIPs closer to industrial applications.

\section{Proto-MIP Concept}

Via the Proto-MIP approach, the necessary selfassembly and hardening/curing steps are separated to generate highly selective cavities for sensing applications.

As can be seen in figure 1 , the (sensor) substrate surface is coated with a fast curing polymeric resin (a), capable to covalently link a suitable anchor group. In parallel the anchor group is introduced to a stamp, containing immobilize target templates/analytes (b). This represents the self-assembly step necessary for MIP generation. Then, the polymeric resin is merged with the anchor group via stamping. In addition, curing (for example via UV irradiation) takes place, representing the necessary hardening step (c). On stamp removal, the desired MIP cavities are left behind.

This separation reduces production time and increases selectivity of the generated artificial receptors. As the polymerization process does not interfere with the previously completed interaction, such as non-covalent binding between functional groups and analyte, of anchor groups with the template surfaces. Separation of the template from the cured MIP layer is carried out by simply taking off the stamp [3]. 


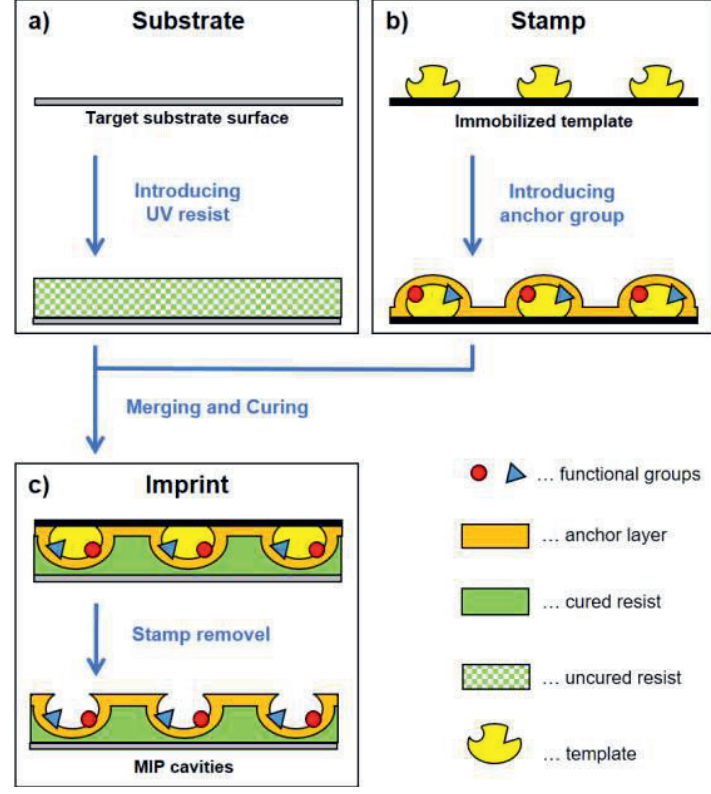

Fig. 1 (a) UV-curable resin is deposited onto a target substrate, (b) stamp with immobilized template is coated with an anchor group, such as functional monomers, (c) merging of polymeric resist and anchor group via polymerization by e.g. UV-exposure resulting in a full MIP layer with imprinted cavities.

\section{Discussion}

The template, in our case E. coli, is immobilized by covalent binding with disuccinimidyl suberate (DSS) on a UV-transmissible polydimethylsiloxane (PDMS) stamp. The functional monomer or so-called anchor group, which consists of a specific composition of the monomers 2-Hydroxyethyl methacrylate (HEMA) and N,N'-Ethylenebis(acrylamide) (EbAM), is coated onto the stamp. After selfassembly, this preliminary MIP layer is fitting chemically as well as sterically the analyte [4]. As the stamp is merged with a thin film of a UVcurable acrylate-based resin, the anchor group has to be compatible to matrices used in nanolithography imprinting.

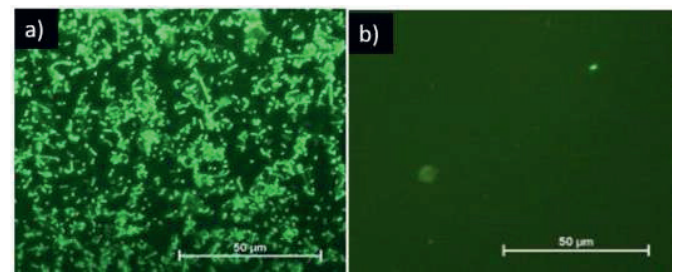

Fig. 2 (a) E.coli covalently bound to stamp surface and labeled with FITC, (b) imprinted polymeric surface revealing no bacteria are sticking to the imprinted surface.

To see whether imprints, without any remaining bacteria on the polymer are obtained, E. coli were stained with FITC [5] before stamp binding as can be seen in figure $2 a$. After removal from imprinted layers, no remaining bacteria species were detectable on the imprinted surface, shown in figure $2 \mathrm{~b}$. In addition, a PDMS stamp with covalently bound $\mathrm{E}$. coli was used to directly imprint the fast UV-curable resin OrmoComp. After removal of the stamp imprints resembling shape and size of the bacteria were detected by AFM measurements as shown in figure $3 a$. Compared to this a much better defined imprint can be seen in figure $3 \mathrm{~b}$, when the anchor group was used for imprinting.

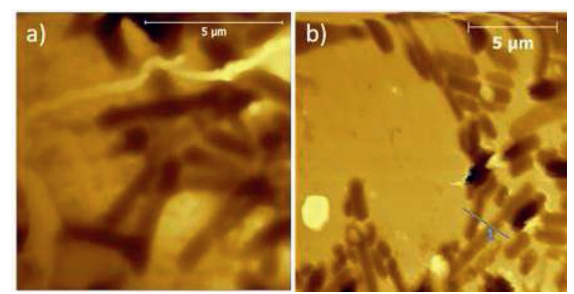

Fig. 3 (a) Immobilized E.coli directly imprinted in UVcurable resin, OrmoComp (OC). (b) anchor group applied onto stamp followed by merging with OC.

\section{Outlook}

Reproducibility and selectivity studies, screening of suitable anchor groups and matrix selection, besides using different templates are ongoing and will be carried out in our future activities.

\section{Acknowledgments}

This research was carried out within the "FTIProject DigiManu - Labor für die Digitalisierung der Produktion" project funded by the State of Upper Austria through the Strategic Economic and Research Program "Innovatives OÖ 2020" and the AquaNOSE project funded by the Austrian Research Promotion Agency (FFG) under Grand Agreement No. 864893. Special thanks to Annette Schnetelker Ph.D., who carried out the first experiments on Proto-MIP and Ao. Univ. Prof. Mag. Dr. Peter Lieberzeit.

\section{References}

[1] R. Schirhagl, Bioapplications for Molecularly Imprinted Polymers, Anal. Chem. 86 (2014) 250261. doi:10.1021/ac401251j.

[2] M. Menger, A. Yarman, J. Erdőssy, H.B. Yildiz, R.E. Gyurcsányi, F.W. Scheller, MIPs and Aptamers for Recognition of Proteins in Biomimetic Sensing, Biosensors. 6 (2016). doi:10.3390/bios6030035.

[3] Profactor $\mathrm{GmbH}$, Verfahren zur Herstellung von Oberflächen mit Affinitätsrezeptoren, Patent Pending, n.d.

[4] SupeIMIP® SPE - Supelco Molecularly Imprinted Polymer SPE Cartridges, Sigma-Aldrich. (n.d.). https://www.sigmaaldrich.com/analyticalchromatography/samplepreparation/spe/supelmip.html (accessed February 26, 2018).

[5] Fluorescein isothiocyanate - an overview | ScienceDirect Topics, (n.d.). https://www.sciencedirect.com/topics/neuroscienc e/fluorescein-isothiocyanate (accessed February 26, 2018). 